\title{
Chilaiditi sign
}

\author{
Haitham Mazek MD
}

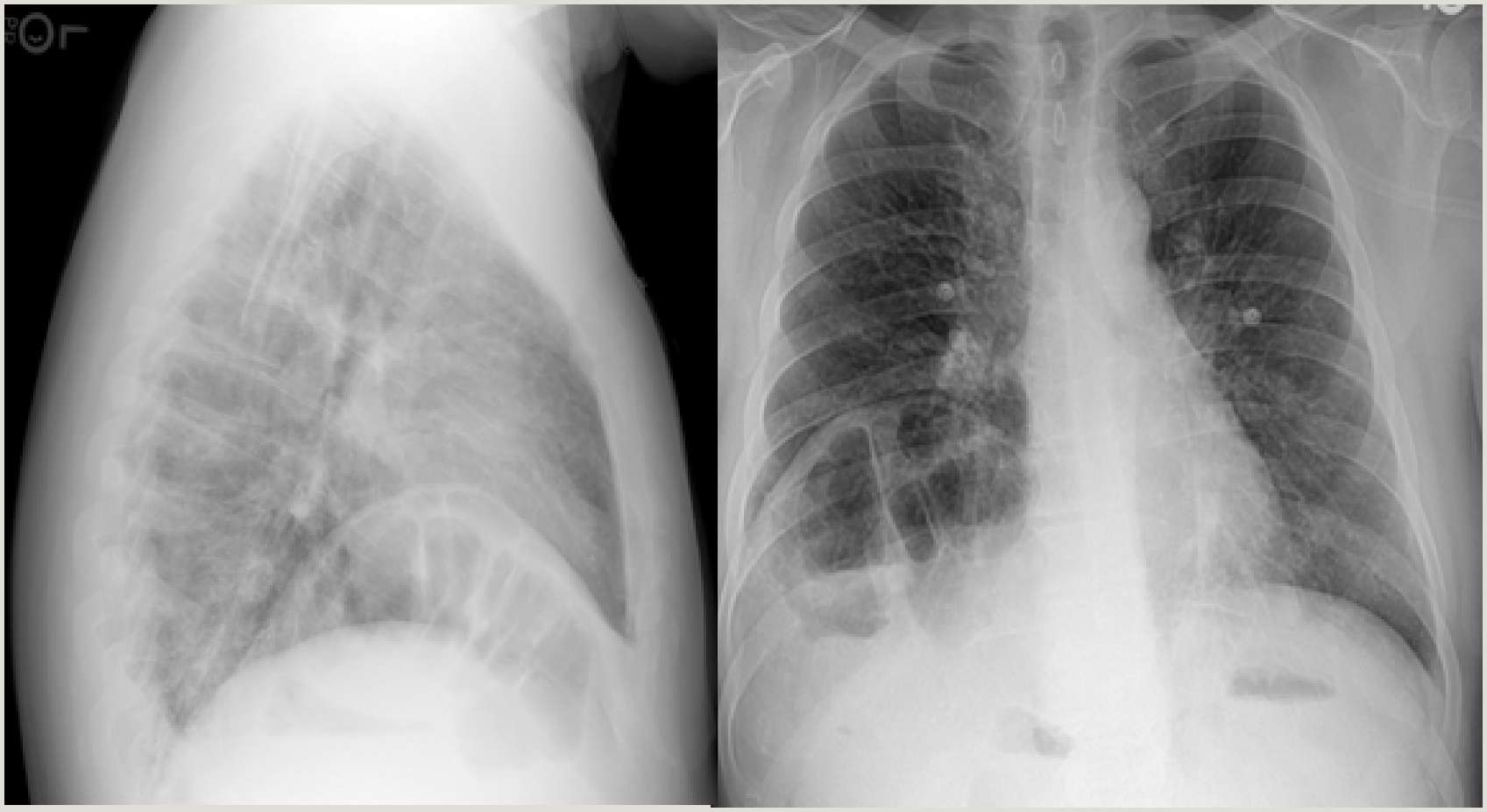

Figure 1. Chest x-ray posteroanterior and lateral showing anterior location of loops of bowel.

Hepatodiaphragmatic interposition of the intestine is known as Chilaiditi sign/syndrome and was described by Demetrius Chilaiditi in 1910. The term "Chilaiditi sign" is used in an asymptomatic person and the term "Chilaiditi syndrome" in symptomatic patients ${ }^{1}$. It is a rare and often asymptomatic anomaly, typically diagnosed as an incidental radiographic finding. However, these patients can have intermittent abdominal pain, distention, vomiting, anorexia, and constipation that on rare occasions require surgical

Corresponding author: Haitham Mazek MD

Contact Information: Haitham.mazek@ttuhsc.edu DOI: 10.12746/swrccc 2014.0207.089 intervention. The prevalence of Chilaiditi's sign in the general population is $0.025-0.28 \%$, and the sign is more common in male patients than female patients ${ }^{2}$.

\section{CASE}

A 65-year-old man presented to the emergency department with worsening shortness of breath, productive cough, subjective fever, and chills for two days. He reported anorexia and fatigue but denied nausea, vomiting, and abdominal pain. On examination, he was afebrile and had normal vital signs. Chest examination revealed decreased air entry into both lower lungs, more so on the right side. His abdomen was mildly distended; no organomegaly and no 
rebound tenderness were detected on palpation. Laboratory studies, including whole blood count, blood chemistry, and urinalysis, were within normal limits. A chest radiograph showed a gas collection under the right hemidiaphragm (Figure 1). His abdominal CT scan demonstrated hepatodiaphragmatic interposition of the colon (Figure 2). The patient treated for community-acquired pneumonia with antibiotics.

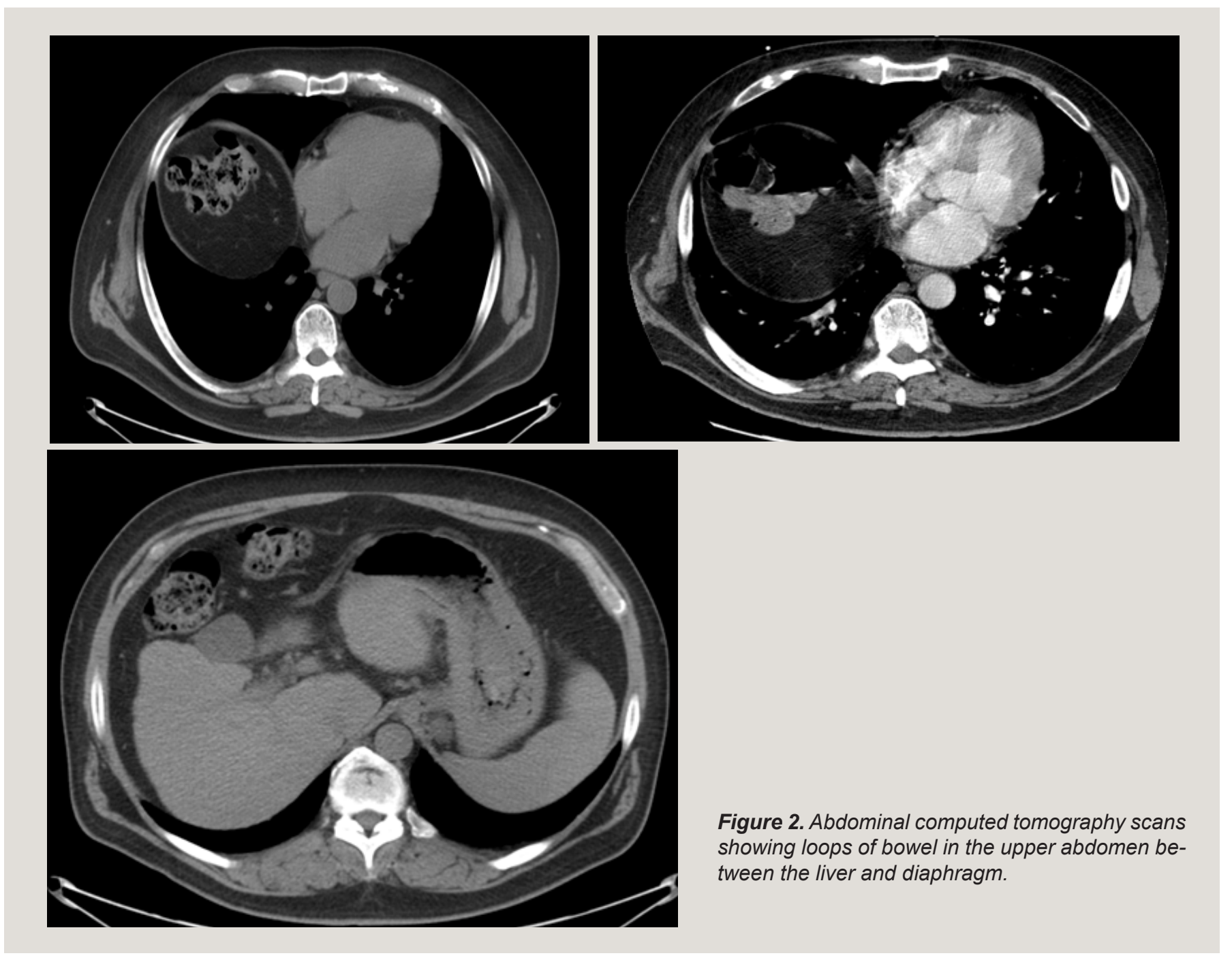

\section{Discussion}

Chilaiditi's sign refers to the presence of a gas filled loop of large bowel in the right upper quadrant between the liver and the right hemidiaphragm. If the patient has gastrointestinal symptoms, then it is referred to as Chilaiditi's syndrome. Predisposing factors for Chilaiditi sign/syndrome are not clear but may include increased colonic mobility, chronic constipa- tion, lax suspensory ligaments, and phrenic nerve injury $^{3}$. It is important to distinguish gas-containing bowel loops between the liver and right hemidiaphragm from other significant pathological conditions, such as perforated viscous, subdiaphragmatic abscess, and pneumoperitoneum, which require surgical operations $^{4}$. In most cases of Chilaiditi syndrome, 
management is conservative and consists of bowel decompression, bowel rest, and aggressive fluid rehydration. Patients who fail conservative therapy should undergo an exploratory laparotomy ${ }^{5}$. Failure of nonsurgical treatment for Chilaiditi syndrome has been associated with colonic volvulus and obstruction ${ }^{6}$.

Author affiliation : Haitham Mazek is a resident in Internal Medicine at TTUHSC.

Submitted: 3/29/2014

Accepted: 6/4/2014

Reviewers: Eman Attaya MD

Conflict of Interest: None

Published electronically: 7/13/2014

\section{References}

1. Murphy JM, Ma1baum A, Alexander G, Dixon AK. Chilaiditi's Syndrome and obesity. Clinical Anatomy 2000; 13:181-4. 2. Choussat H, Choussat-Clausse J. Deux cas d'interposition hepatodiaphragmaticdu colon avec autopsies. Bulletin et Memoires de la Societe de Radiologie Medicale de France 1937; 25:147-154.

3. Chang TY, Tiu CM, Chou YH, Huang LL, Yu C. Hepatodiaphragmatic interposition of the intestine: Chilaiditi's Syndrome. Chin J Radiol 1999; 24:101-5.

4. Havenstrite KA, Harris JA, Rivera DE. Splenic flexure volvulus in association with Chilaiditi syndrome: report of a case. Amer Surg 1999; 65(9):874-6.

5. Kurt Y, Demirbas S, Bilgin G, et al. Colonic volvulus associated with Chilaiditi's syndrome: report of a case. Surg Today 2004; 34:613-615.

6. Orangio GR, Fazio VW, Winkelman E, McGonagle BA. The Chilaiditi syndrome and associated volvulus of the transverse colon. an indication for surgical therapy. Dis Colon Rectum $1986 ; 29: 653-656$. 